\title{
Dipole and Mass Formation Are Two Phenomena Causing Formation of Particle and Antiparticle in Pair Production Phenomena
}

\author{
S.H. Dhobi ${ }^{*}$, M.D.J. Rangrej $^{2}$, G.P. Adhikari ${ }^{3}$, M.N. Upadhyay ${ }^{4}$ \\ ${ }^{1,2,3,4}$ Department of Physics, Tribhuvan University , Kathmandu-44600, Nepal \\ ${ }^{1,2,3,4}$ Innovative Ghar Nepal Laboratory, Innovative Ghar Nepal, Lalitipur-44700, Nepal \\ *Corresponding Author: saddam@ran.edu.np, Tel.: +977-9808100285
}

Available online at: www.isroset.org

Received: 23/Nov/2019, Accepted: 11/Dec/2019, Online: 31/Dec/2019

\begin{abstract}
The framework of this work is to show, the phenomena of formation of particle and antiparticle in pair production when energy of suitable photon incidence or reach to positive nucleus field of an atom. In this phenomena before the formation of antiparticle and particle the energy of photon is converted into mass and due to charge of nucleus this formation mass goes on dipole formation, such formation is take place in weak positive nucleus filed. After the formation of dipole the orientation of positive part of mass is away from nucleus central field and negative part is towards the center of positive nucleus field. On going the formation mass with dipole towards the strong nucleus field, the repulsive force between of positive charge of mass i.e. antiparticle formation part, and positive charge nucleus is greater than that of attractive force of antiparticle part and particle part of mass having dipole, formed from incidence photon. Moreover, the formation of antiparticle is first, take place at the boundary of weak nucleus field and strong nucleus field. After the formation of antiparticle formation of particle part is separated and goes on repulsion on repulsive nucleus field.

Hence in this way we can explain the phenomena of formation of particle and antiparticle in pair production and leaving from atom or positive charge field of nucleus.
\end{abstract}

Keywords - Suitable photon, Formation of mass, Weak nucleus field, Strong nucleus field, Repulsive nuclear field, particle and antiparticle etc.

Note: Particle is negatively charge and antiparticle is positively charge in our assumption.

\section{INTRODUCTION}

In high electric field, electrons become runaway if the accelerating force is higher than the drag force due to collision of electrons with air. The electron runaway mechanism, in which electrons may accelerate in static electric fields in air, this phenomena was first described by Wilson [1]. If we approximate the problem in which all protons are located at the center of the nucleus then from Biot-Savart law we get an expression and value,

$-\mathrm{eBy} \sim 2 \mathrm{Z}_{\mathrm{Au}} \gamma\left(\mathrm{e}^{2} / 4 \pi\right) \mathrm{v}_{\mathrm{z}}(2 / \mathrm{b})^{2} \approx 10 \mathrm{~m}_{\pi}^{2} \approx 10^{19}$ Gauss,

...............(1)

where $\mathrm{v}_{\mathrm{z}}=\sqrt{ }\left(1-\left(2 \mathrm{mN} / \sqrt{\mathrm{s}}^{2}\right) \approx 0.99995\right.$ (here, $\mathrm{mN}$ is the nucleon mass $)$ is the velocity of the nucleus, $\gamma=\sqrt{ }\left(1-\mathrm{v}_{\mathrm{z}}^{2} \approx\right.$ 100 is the Lorentz gamma factor, and $\mathrm{Z}_{\mathrm{Au}}=79$ is the charge number of gold nucleus. The minus sign indicate magnetic field in pointing to the $-y^{\wedge}$ direction.

Also,

$$
\begin{aligned}
& e E(t, r)=\frac{e^{2}}{4 \pi} \sum_{n} Z_{n} \frac{\left(R_{n}^{\prime}-R_{n} v_{n}\right)}{\left(R_{n}-R_{n}^{\prime} v_{n}\right)^{3}}\left(1-v_{n}^{2}\right) . \\
& e B(t, r)=\frac{e^{2}}{4 \pi} \sum_{n} Z_{n} \frac{\left(v_{n} \times R_{n}^{\prime}\right)}{\left(R_{n}-R_{n}^{\prime} v_{n}\right)^{3}}\left(1-v_{n}^{2}\right) \ldots .
\end{aligned}
$$

where the summation is over all the charged particles, $\mathrm{Zn}$ is the charge number of the $n^{\text {th }}$ particle, $R_{n}=r-r_{n}$ is the relative position of the field point $r$ to the source point $r_{n}$ of the $n^{\text {th }}$ particle, $v_{n}$ is the velocity of $n^{\text {th }}$ particle at the retarded time $t_{n}=t-\left|r-r_{n}\right|$. Note that Eqs. (2)-(3) have singularities at $R_{n}=0$. EM fields in heavy-ion collisions obtained by using the Li'enardWiechert potentials on event-by-event basis example $\mathrm{Au}+\mathrm{Au}$ collisions at RHIC and $\mathrm{Pb}+\mathrm{Pb}$ collisions at LHC [2].

The process of direct electron-positron pair production via the interaction of energetic runaway electrons with atomic nuclei. As the direct positrons are produced by runaway electrons with no photon mediators, their initial distribution in the transverse direction with respect to the axis of primary avalanche plane. Therefore, when these positrons are eventually turned around by electric field and become the 
runaway, secondary electron avalanches are initiated with them predominantly close to primary avalanche axis [3].

The cross section of the direct formation of electron-positron pairs by electrons in the field of a nucleus, in the relativistic limit, is calculated by Bhabha using the relation

$\sigma_{e^{-} \rightarrow e^{-} e^{-} e^{j e f}}=\frac{28 Z^{2} r_{e}^{2} \alpha^{2}}{27 \pi} \ln ^{3}\left(\frac{E_{0}}{m c^{2}}\right)$

where $\mathrm{Z}$ indicate charge of nuclei, re indicate classical electron radius, $\square$ indicate fine structure constant, $\mathrm{E}_{0}$ indicate total incident electron energy, and $\mathrm{m}$ indicate electron mass [4].

The cross section of process of equation (1) near the threshold is calculated by Gryaznykh using the relation

$$
\sigma_{e^{-} \rightarrow e^{-} e^{-} e^{+}}=\frac{7 Z^{2} r_{e}^{2} \alpha^{2}}{2304} \frac{\left(T_{0}-2 m c^{2}\right)^{3}}{\left(m c^{2}\right)^{3}}
$$

where $\mathrm{T0}$ is the incident electron kinetic energy [5].

The $\mathrm{CMB}$ has a blackbody spectrum with $\mathrm{T}=2.7 \mathrm{~K}$ corresponding to mean energy of $6.34 \times 10^{-4} \mathrm{eV}$. A proton of high enough energy $\left(>7 \times 10^{19} \mathrm{eV}\right)$ will interact inelastically with $\mathrm{CMB}$ photons producing pions. The reaction are given

$$
\begin{gathered}
\gamma+P \rightarrow n+\pi^{+} \\
y+P \rightarrow n+\pi^{0}
\end{gathered}
$$

In accordance with the theory of relativity the kinetic energy of a material point of mass $m$ is express as

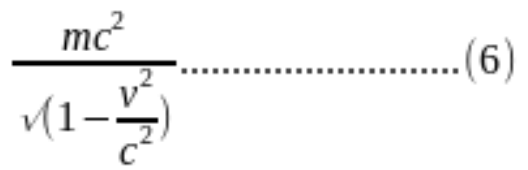

instead $\mathrm{mv}^{2} / 2$. This expression approaches infinity as the velocity $\mathrm{v}$ approaches the velocity of light $\mathrm{c}$. The velocity must therefore always remain less than $\mathrm{c}$.

If we develop the expression for the kinetic energy in the form of a series, we obtain

$$
m c^{2}+m \frac{v^{2}}{2}+\frac{3}{8} m \frac{v^{4}}{c^{2}}+
$$

when $\mathrm{v}^{2} / \mathrm{c}^{2}$ is small compared with unity, the third of these terms is always small in comparison with the second, which last is alone considered in classical mechanics Relativistic mass of body or particle can be express as

[6].

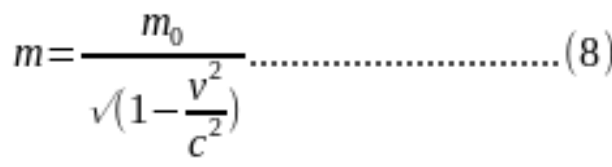

where,

$\mathrm{m}_{0}$ indicate rest mass and $\mathrm{m}$ indicate mass a any instant $[7,8,9]$.

\section{RELATED WORK}

Theoretical and Experimental knowledge of electronpositron pair production by photons, from the prediction of the positron by Dirac. Photons of energies above $2 \mathrm{~m}_{\mathrm{e}} \mathrm{c}^{2}$ $(1.022 \mathrm{MeV})$ can interact with the Coulomb field of an atomic nucleus to be transformed into an electron-positron pair. This interaction can also take place in the field of an atomic electron, for photons of energy in excess of $4 \mathrm{~m}_{\mathrm{e}} \mathrm{c}^{2}$ $(2.044 \mathrm{MeV})$, in which case the process is called triplet production.

Pair and triplet cross sections are predominant contributions to the photon mass attenuation coefficient for photon energies $10 \mathrm{MeV}$ and higher. Hence, for pair production in the field of a nucleus, the cross section $k_{n}$ per atom is $k_{n} \alpha Z^{2}$ in which $\mathrm{Z}$ is the atomic number, or proton number, of the target nucleus. For triplet production, for which the target electron has unit charge, the cross section $k_{\mathrm{e}}$ for a neutral atom with $\mathrm{Z}$ electrons is $\mathrm{k}_{\mathrm{e}} \alpha \mathrm{Z}$ and thus $\mathrm{k}_{\mathrm{e}} / \mathrm{k}_{\mathrm{n}} \simeq 1 / \mathrm{Z}$ for incident photon energies well above the differing thresholds [10].

The magnetic field created in proton-proton and nucleusnucleus collisions at ultra-high energies. The magnetic-field strength immediately after collisions reaches the value tens of $\mathrm{GeV}^{2}$. The absolute value of the magnetic field rapidly decreases with time and increases with growth of atomic number. The amplitude for eB is estimated at level $100 \mathrm{GeV}^{2}$ to provide magnitude for quark-quark collisions at energies corresponding to the nominal energies of proton beams [11].

Two positively charged heavy nuclei produce ultra-intense magnetic fields in collider experiments at the Relativistic Heavy Ion Collider (RHIC) and at the Large Hadron Collider (LHC), e.g. $\mathrm{B} \sim 10^{18}-10^{19} \mathrm{G}$ for $\sqrt{\mathrm{sNN}}_{\mathrm{s}}=200 \mathrm{GeV} \mathrm{Au}+\mathrm{Au}$ collisions. We use natural units $\sim \hbar=c=\epsilon_{0}=\mu_{0}=1$, where $\epsilon_{0}$ and $\mu_{0}$ are the electric permittivity and magnetic permeability in vacuum, respectively, and the electric charge $\mathrm{e}:=\sqrt{ }(4 \pi \hbar c \alpha) \sim 0.303$, where $\alpha=1 / 137$ is the fine-structure constant [12]. In high energy heavy ion collisions positively charged nucleus collide at a speed of light and produce intense magnetic fields. Field rapidly fall off as we move away from charge along $\mathrm{z}$ or away from nucleus [13].

Rutherford blasted alpha particles at a thin piece of gold foil, in order to get the positively charged alpha particle near to the nucleus of a gold atom, high energies were needed to overcome the electrostatic force of repulsion. After this 
energy is re-distributed producing new particles. The higher the collision energy the larger the mass of the particles that can be produced and there will be no energy left over for the creation of new particles [14]. In a relativistic heavy-ion collision, electromagnetic field is created by $\mathrm{Z}$ electric charges of one ion and $\mathrm{Z}$ electric charges of another ion moving in the opposite directions along, say, $\mathrm{z}$ axis and total classical field is a sum of fields of all charges, superposition principle [15].

The complexity of particle-matter interactions is an of highenergy protons: if high-energy protons $(\mathrm{GeV})$ impact on a material, they are subject to inelastic nuclear collisions. Particle beams in a high-energy collider, like LHC, with a stored energy of hundreds of MJ, have an enormous damage potential. Higher photon energies, pair production in the field of nuclei is the dominating absorption mechanism of photons. The threshold energy for electron-positron pair production is $1.022 \mathrm{MeV}$, i.e., the combined rest energy of an electron and a positron. The cross-section scales approximately with Z2. The energy above which pair production dominates is less for higher- $\mathrm{Z}$ materials (around $25 \mathrm{MeV}$ for carbon and around $5 \mathrm{MeV}$ for lead). The differential cross-section for an energy transfer $\mathrm{T}$ to an electron, then the mean energy loss per unit path length, which is generally referred to as the electronic stopping

$$
\frac{d E}{d x} / \text { elec }=N \int_{0}^{T_{0 \mathrm{max}}} T \frac{d \sigma}{d T}(T) d T
$$

power, is given by

(photon loses energy with approaches to nucleus ) where Tmax is the maximum energy transfer. The mean free path

$$
\sigma_{\text {pair }} \approx \frac{7}{9} \frac{M}{\rho N_{A} X_{0}} \approx \frac{7}{9} X_{0} \begin{aligned}
& \text { are constant at hitten as } \\
& \text { energies and can be }
\end{aligned}
$$

where $\mathrm{M}$ indicate molar mass and $\rho$ indicate material density, respectively. This means that the radiation length is about 7/9 of the mean free path of high-energy photons and therefore determines the photon survival probability can be written as,

$$
\begin{gathered}
I(x)=I_{00} \cdot \exp \left(\frac{-7}{9} \frac{x}{X_{0}}\right) \\
X_{0}^{\prime}=\frac{716.4 \mathrm{gm} / \mathrm{cm}^{3} \mathrm{~A}}{\mathrm{Z}(\mathrm{Z}+1) \ln (287 / \sqrt{\mathrm{Z}})}
\end{gathered}
$$

where $X^{\prime}{ }_{0}$ is $X^{0} \rho$ in $g / c m 2$, and $Z$ and $A$ are the atomic and mass numbers, respectively [16].
Nuclei consist of nucleons which in turn consist of quarks and gluons, photons consist of lepton pairs, electrons consist of photons, etc. Relativistic nuclei have photons as an important constituent, especially for low enough virtuality, $\mathrm{Q}^{2}=-\mathrm{q}^{2}>0$ of the photon. This is due to the coherent action of all the charges in the nucleus. The equivalent photon spectrum extends up to several $\mathrm{GeV}$ at RHIC energies $(\gamma \approx$ $100)$ and up to about $100 \mathrm{GeV}$ at LHC energies $(\gamma \approx 3000)$.

Atom is almost empty for gamma rays photon: interaction between electron, nucleus with gamma rays is very low in the energy range from some hundreds of $\mathrm{eV}$ to several $\mathrm{MeV}$ (E <10 MeV). Energy range between roughly $10 \mathrm{MeV}$ and $20 \mathrm{MeV}$ a bump due to the decay of the Giant Dipole Resonance (GDR). Energy range beyond $30 \mathrm{MeV}$ the spectrum is characterized by a large inverse slope parameter which increase with increasing energy and with a yield which, for a given incident energy per nucleon, increases with the size of the colliding nuclei. These high energy gamma rays $(\mathrm{E}>30 \mathrm{MeV})$ are the so called hard photons [17].

Photon-orbital electron interactions are characterized as interactions as a loosely bound electron i.e. Compton effect, triplet production, a tightly bound electron i.e. photoelectric effect. The interactions of photons with nuclei as Direct photon-nucleus interactions i.e. photodisintegration, Interactions between the photon and the electrostatic field of the nucleus i.e. pair production. When photon interact with nucleus electromagnetic field it transfer it whole energy and only causes of pair production [18].

The range of the nuclear force is short, only a few femtometer $\left(1 \mathrm{fm}=10^{-15} \mathrm{~m}\right)$, beyond which it decreases rapidly and is due exchange of particles lighter than nucleons known as mesons. Scattering experiments at higher energies more than $200 \mathrm{MeV}$, provide evidence that the nucleonnucleon interaction turns repulsive at short inter-nucleon distances when smaller than $0.5 \mathrm{fm}$, but it rapidly decreases to insignificance at distances beyond about $2.5 \mathrm{fm}$ [19].

\section{MATERIALS AND METHODS}

Let us consider $\mathrm{M}$ is the mass of moving photon having suitable energy hf for pair production, where $h$ is plank constant and $\mathrm{f}$ is frequency of same consider photon frequency. Also let $M_{R}$ is the rest energy of photon with energy $M_{R} c^{2}$, where $c$ is velocity of light and.

Then from Einstein relativity theory, we have

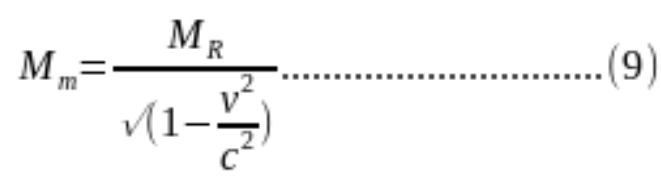


Here $M_{m}$ is the mass of photon in moving state or position and $\mathrm{MR}$ is the mass of photon at rest position or state and energy

$$
E=\frac{M_{0} c^{2}}{\square\left(1-\frac{v^{2}}{c^{2}}\right)} \ldots \ldots \ldots \ldots \ldots \ldots \ldots \ldots(10)
$$

In our consideration the pair production is occur in two step i.e. before the pair production the energy of incidence photon in nucleus field converted into mass and then mass is converted into particle of opposite charge due to nucleus field. The following two step are given below:

\section{A. Step First: First the energy of incidence convert into mass}

In this the energy is converted into a single concrete mass because as the photon of suitable energy incidence or going to closure in nucleus field the field repel the field of incidence photon and hence the velocity of photon goes to decreases. This follow the relation (9) and let $v$ velocity of incidence photon become $\mathrm{v}_{\mathrm{f}}$ after entering on nucleus field then from equation (9), $\mathrm{M}$ become $\mathrm{M}_{\mathrm{f}}$ is mass of photon after

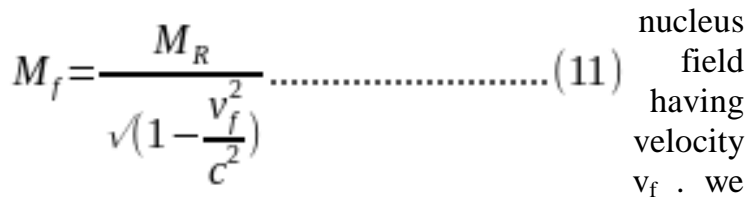

have

[Photon at rest in nucleus field]

Here, $v_{f}<c$ then that of $v<c$ from (9) because the velocity is going to decreases as photon in approaching to nucleus field.

Finally the the velocity of photon in nucleus field goes to zero $\left(v_{\mathrm{f}}=0\right.$ i.e. rest) then from equation (11) we have,

$$
M_{f R}=\frac{M_{R}}{\sqrt{ }\left(1-\frac{0}{c^{2}}\right)}
$$

$\mathrm{M}_{\mathrm{fR}}$ indicate the mass of photon at rest inside nucleus field.

$$
M_{F R}=M_{R}
$$

This an equation that show photon at a point in nucleus field get at rest with mass $\mathrm{M}_{\mathrm{fR}}$ or $\mathrm{M}_{\mathrm{R}}$.

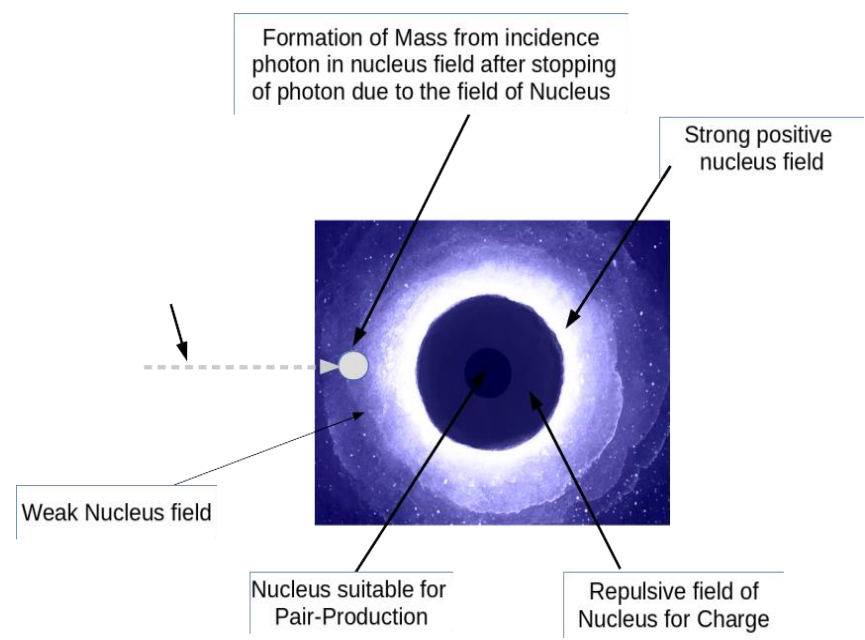

Figure 1: Formation of mass in weak positive nucleus field in atom, in pair-production.

This is such mass which is different from ordinary mass and its composition i.e. different from ordinary mass composition like quark, electron, proton, neutron. Because this mass formed only positive and negative particle from incidence photon which is suitable for pair production.

\section{B. Step Second: Second mass get ionized into two or more particle having opposite charge}

After formation mass $\mathrm{M}_{\mathrm{fR}}$ in nucleus field. The formation of mass concentration is in such a way that the negative charge concentration toward nucleus field center and positive charge concentration are away from center of nucleus field. It is because nucleus center is the concentration of positive charge particle and create positive nucleus field around the nucleus i.e. protons is positive charge particle and neutrons neutral charge so the effect of proton cause positive nucleus field around nucleus.

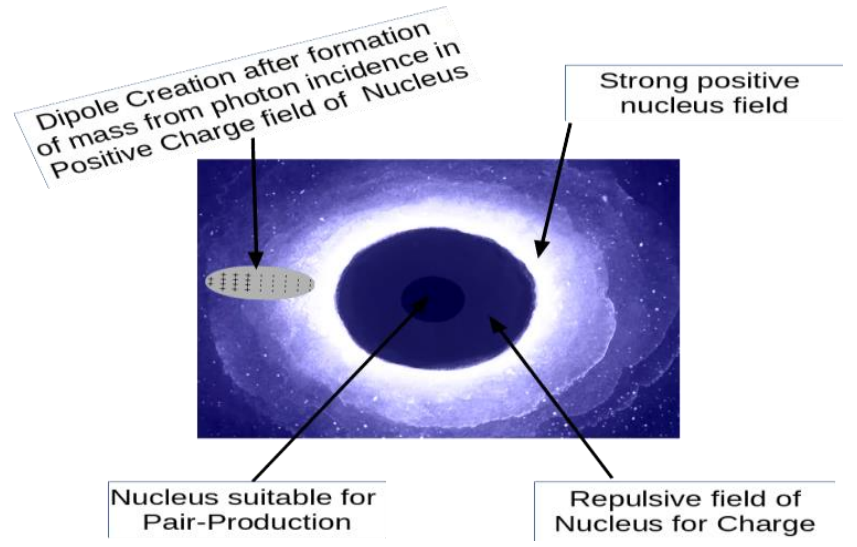

Figure 2: Formation of dipole momentum in mass formed in pair-production. 
Two separated charges of opposite sign, $\mathrm{q}_{1}=-\mathrm{q}$ and $\mathrm{q}_{2}=+\mathrm{q}$ form an electric dipole. The dipole moment is defined by:

$$
\mu^{\square}=q\left(r_{2}^{\square}-r_{1}^{\square}\right)=q r^{\square}
$$

where $r_{1}^{\square}$ and $r_{2}^{\square}$ are the vectors that define the position of the two charges in space. The dipole moment is thus a vector quantity. For a distribution of negative and positive point charges $\mathrm{q}_{\mathrm{i}}$ the dipole moment is:

$$
\mu \vec{\mu}=\sum_{i} q_{i} r_{i}^{\vec{i}} \text {. }
$$

where $r_{i}^{\square}$ are the positions of the charges $q_{i}$ [20].

In Figure 2 we can seen that after the formation of mass in boundary of weak and strong positive nucleus field the mass goes on electric dipole due to the presence of positive and high nucleus field. The positive charge of mass is directed away from the proton field of nucleus and negative charge towards the proton field of nucleus or repulsive nucleus field, which is shown in figure 2 .

After the formation of positive and negative charge due to the field of nucleus, on formation from incidence photon. After the formation of charge they are attracted with coulomb force

$$
F=K \frac{q_{1} q_{2}}{r^{2}}
$$

where $\mathrm{K}$ is coulomb constant, $\mathrm{q}_{1}$ is positive charge and $\mathrm{q}_{2}$ is negative charge separated by distance $r$.

This coulomb force hold the positive and negative in or at boundary of weak and strong nucleus field for a certain time after the formation of mass and charge. But after some time as dipole formation mass moves towards repulsive nucleus field and due to repulsive force generated between positive charge of mass and nucleus proton, positive part of mass separated from negative i.e. repulsive force overcome attractive force. The sepration of positive charge and negative charge of mass are shown in figure 3.

Figure 3 is showing how the separation of positive and negative charge of formed mass from photon energy. In figure 3 red part indicate positive charge or antiparticle, which is in weak positive nucleus field and, yellow part is the collection of negative charge indicate particle in strong nucleus field and slightly in repulsive nucleus field. This indicate that the formation of antiparticle is first and then after certain time particle is formed in pair production phenomena.

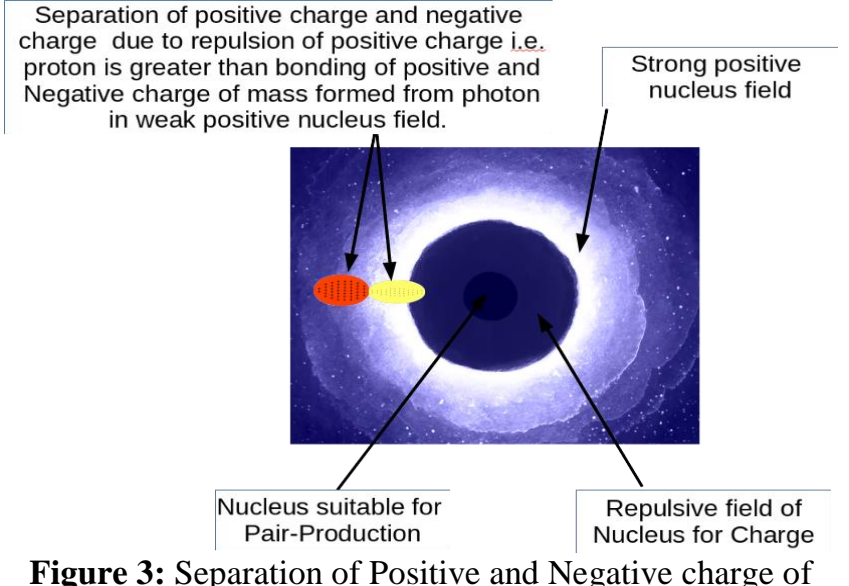

Figure 3: Separation of Positive and Negative charge of mass due to positive nucleus field.

Figure 4 indicate the escaping of the particle and antiparticle with few time in-travel between them. In this figure due to the repulsion of positive charge particle particle in nucleus field, positive escape from the weak positive nucleus field and negative charge escape from repulsive nucleus field. Here repulsive nucleus field repel the charge particle away, which is negative and due to this negative charge particle escape out from nucleus field and hence show the formation of particle and antiparticle.

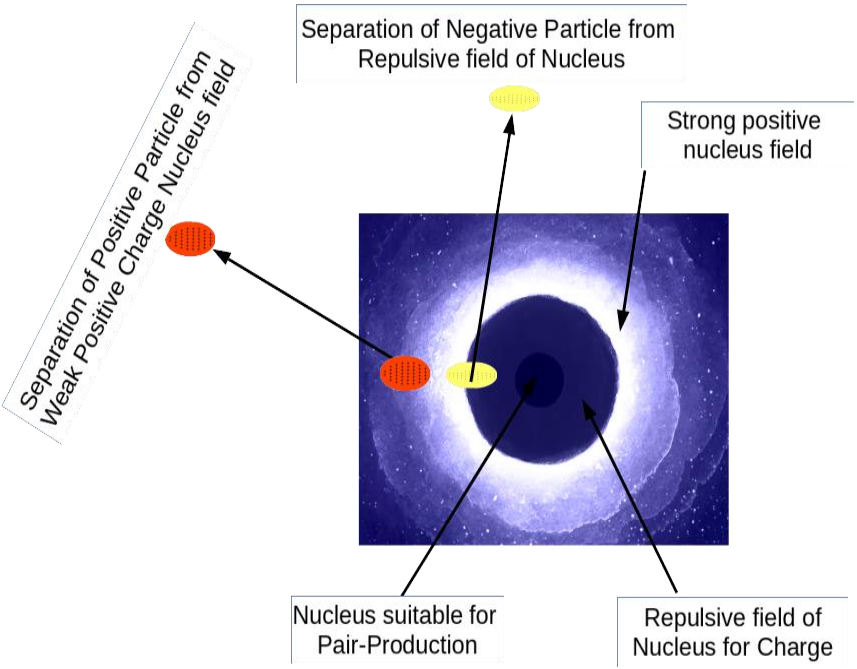

Figure 4: Separation or formation of positive particle particle and negative charge particle and ejection from difference field of nucleus.

\section{RESULTS AND DISCUSSION}

Pair-production is the phenomena that take place in two steps, in first step the formation of mass take place in or at the boundary of weak and strong positive nucleus field. Formation of mass follow the principle of energy-mass i.e. energy of incidence photon converted into mass due the decreasing of photon velocity in positive nucleus field of 
consider nucleus. After the formation of mass, second step start and in this step electric dipole take place on the mass formed in first step. The electric dipole formation on mass take place due to positive nucleus field. After formation of electric dipole on mass, the positive and negative charge particle goes on separation because the coulomb attraction force on mass due to electric dipole is overcome the repulsion force created between positive part of mass and positive charge of proton in nucleus field. Due to this repulsion force the positive part of mass is separated called antiparticle with positive charge and escape away from the nucleus. The remain negative part of mass i.e. negative charge particle are attracted towards the positively charge proton and when negative part enter in the repulsive region of nucleus field the field repel the negative charge particle instead of attraction and hence particle with negative charge escape from nucleus field. This show that positive charge particle form mass formed in nucleus field created first and then negatively charge particle, after certain time.

\section{CONCLUSION AND FUTURE SCOPE}

In this paper we calculated or generated idea to show how the formation of particle and antiparticle take place in pairproduction and which particles formed first and which formed later. On solving and studying we find that pairproduction take place in two step i.e. first energy is converted into mass and then electric dipole is centered in mass in nucleus field, and after formation of electric dipole the positive and negative charge part of mass goes to separated. After the separation of positive and negative charge of mass this goes on apart from each other due to the different strength of nucleus field. This show that the formation of particle and antiparticle take time in-travel i.e. particle and antiparticle doesn't formed at instant, at instant they goes apart and after apart due to different strength of nucleus field one escape first and then other escape.

\section{ACKNOWLEDGMENT}

We would like to hanks all members of Innovative Ghar Nepal, Lalitipur-44700, Nepal for provide research space and peaceful environment during our research work.

\section{REFERENCES}

[1] C. Wilson, "The acceleration of $\square$-particles in strong electric fields such as those of thunderclouds", Math. Proc. Cambridge Philos. Soc., vol. 22, Issue 4, pp.534-538, 1925.

[2] X.G. Huang, "Electromagnetic fields and anomalous transports in heavy-ion collisions: A pedagogical review", pp.4-6, 9 June, 2016.

[3] I.B. Vodopiyanov, J.R. Dwyer, E.S. Cramer, R.J. Lucia, and H. K. Rassoul, "The effect of direct electron-positron pair production on relativistic feedback rates, Journal of Geophysical Research: Space Physics, Vol. 120, pp.805-806, 2015.
[4] H.J. Bhabha, "The creation of electron pairs by fast charged particles", Proc. R. Soc. London, Ser. A, Vol. 152, pp.559586, 1935.

[5] D.A. Gryaznykh, "Cross section for the production of electronpositron pairs by electrons in the field of a nucleus", Phys. At. Nucl., Vol. 61, No.3, pp.394-399, 1998.

[6] A. Einstein, "Relativity The Special And General Theory, New York: Henry Holt And Company," pp.53-54, 1920.

[7] J.D. Cresser, "The Special Theory Of Relativity: Lecture Notes Prepared”, Macquarie University, Sydeny, pp.29, July 31, 2003.

[8] J. D. Cresser, "Lecture Notes On Special Relativity", Macquarie University Sydney, pp.38, August 18, 2005.

[9] P. Harris, "Lecture Note: Special Relativity", University of Sussex, pp.67-68.

[10] J.H. Hubbell, "Electron-positron pair production by photons: A historical overview", Radiation Physics and Chemistry, Vol.75, pp.614-615, 2006.

[11] V.A. Okorokov, "Magnetic Field in Nuclear Collisions at Ultra High Energies", Physics 2019, Vol.1, pp.183, 2019.

[12] V. Roy, S. Pu, L. Rezzolla, D.H. Rischke, "Effect of intense magnetic fields on reduced-MHD evolution in ${\sqrt{\mathrm{S}_{\mathrm{NN}}}}=200$ $\mathrm{GeV} \mathrm{Au}+\mathrm{Au}$ collisions, pp.2, ${ }^{\text {th }}$ Dec 2017.

[13] V. Roy, "Importance of Magnetic Field on Hydrodynamics evolution in Heavy Ion Collisions", J W Goethe University, Frankfurt am Main, Germany, p3-6. [presentation].

[14] S. Lucas, "Particle Accelerators", University College London, p228, Presentation.

[15] K. Tuchin, "Initial value problem for magnetic field in heavy ion collisions", p.2, 7 October 2018.

[16] A. Lechner, "Particle Interactions with Matter," CERN Yellow Reports: School Proceedings, Vol. 5, pp.47-57,. Edited by B. Holzer. 2018

[17] A. Bonasera, R. Coniglione and P. Sapienza, "High Energy Probes", INFN - Laboratori Nazionali del Sud, Via S. Sofia 62, 95123 Catania Italy, p.8.

[18] D. Adliené, "2. Radiation interaction with condensed matter", Department of Physics Kaunas University of Technology, Presentation. pp. 43-70, Oct. 2017.

[19] R. Machleidt, "Nuclear Forces," Schola., Vol. 9, No. 1, pp.30710, 2014, Revision: 2019.

[20] Physikalisch-chemisches Praktikum I, Dipole Moment, p1-2, 2016. 